\title{
Automatic Grade Classification of Barretts Esophagus through Feature Enhancement
}

\author{
Noha Ghatwary ${ }^{\mathrm{a}, \mathrm{b}}$, Amr Ahmed $^{\mathrm{a}}$, Xujiong Ye ${ }^{\mathrm{a}}$, and Hamid Jalab \\ ${ }^{a}$ University of Lincoln, Lincoln, United Kingdom \\ ${ }^{\mathrm{b}}$ Arab Academy for Science and Technology, Alexandria, Egypt \\ ${ }^{\mathrm{c}}$ University of Malaya,Kuala Lumpur, Malaysia
}

\begin{abstract}
Barretts Esophagus (BE) is a precancerous condition that affects the esophagus tube and has the risk of developing esophageal adenocarcinoma. $\mathrm{BE}$ is the process of developing metaplastic intestinal epithelium and replacing the normal cells in the esophageal area. The detection of BE is considered difficult due to its appearance and properties. The diagnosis is usually done through both endoscopy and biopsy. Recently, Computer Aided Diagnosis systems have been developed to support physicians opinion when facing difficulty in detection/classification in different types of diseases. In this paper, an automatic classification of Barretts Esophagus condition is introduced. The presented method enhances the internal features of a Confocal Laser Endomicroscopy (CLE) image by utilizing a proposed enhancement filter. This filter depends on fractional differentiation and integration that improve the features in the discrete wavelet transform of an image. Later on, various features are extracted from each enhanced image on different levels for the multi-classification process. Our approach is validated on a dataset that consists of a group of 32 patients with 262 images with different histology grades. The experimental results demonstrated the efficiency of the proposed technique. Our method helps clinicians for more accurate classification. This potentially helps to reduce the need for biopsies needed for diagnosis, facilitate the regular monitoring of treatment/development of the patients case and can help train doctors with the new endoscopy technology. The accurate automatic classification is particularly important for the Intestinal Metaplasia (IM) type, which could turn into deadly cancerous. Hence, this work contributes to automatic classification that facilitates early intervention/treatment and decreasing biopsy samples needed.
\end{abstract}

Keywords: Barretts Esophagus, Fractional Differentiation, Fractional Integration, Confocal Laser Endomicroscopy

\section{INTRODUCTION}

Esophageal cancer is considered one of the main causes of the increased death rate in industrial countries, due to the difficulties of early detection and diagnosis. ${ }^{1}$ It is considered the top 18th cancer worldwide with a 5year survival rate plan of $18.2 \%$ only. Barretts Esophagus (BE) is the main precancerous condition that affects the lower cells of the esophagus columnar. ${ }^{2}$ Patients with BE are considered at high risk to have esophageal adenocarcinoma in later stages, it started to spread widely especially in western countries due to several reasons such as fast food, factories, smoking etc... affecting for 1.6 to 6.8 percent of the population. ${ }^{3}$ It usually has no symptoms but except for patients that are already diagnosed with Gastroesophageal Reflux Disease (GERD) that might be a predevelopment case, ${ }^{4}$ where around 10\%-15\% GERD patients can have the risk that the esophagus lining turns to BE. The detection of BE needs to be done through an endoscopy examination, the physician needs to be an expert and well trained to the type of endoscopy used in diagnosis to be able to detect the lesion area. Biopsy also is required to confirm the diagnosis of the BE. ${ }^{3}$

Computer aided diagnose (CAD) can help doctors in the second opinion and decrease the number of biopsies needed for detection and classification. ${ }^{5}$ It is one of the popular systems designed utilizing computer vision and

Further author information: (Send correspondence to N.G,A.A)

N.G: E-mail: nghatwary@lincoln.ac.uk, noha.ghatwary@aast.edu

A.A.: E-mail: aahmed@lincoln.ac.uk

Medical Imaging 2017: Computer-Aided Diagnosis, edited by Samuel G. Armato III, Nicholas A. Petrick, Proc. of SPIE Vol. 10134, 1013433 - @ 2017 SPIE · CCC code: 1605-7422/17/\$18 · doi: 10.1117/12.2250364 
image processing to assist doctors in analyzing the medical images. ${ }^{6}$ The typical CAD system passes through a variety of phases as shown below in figure 1. A Medical Video Frame or Image is first exposed to pre-processing phase through enhancement and noise reduction to provide a clearer view of some image-hidden details and regions. Eventually, the targeted organ (if required) is segmented and extracted from the medical image. By working on the extracted organ itself or the cells, the detection stage focuses on disclosing abnormal areas and lesions detection. Finally, the segmented/detected lesions are classified based on extracted improved features into the relevant cancer type or severity, in the classification stage.

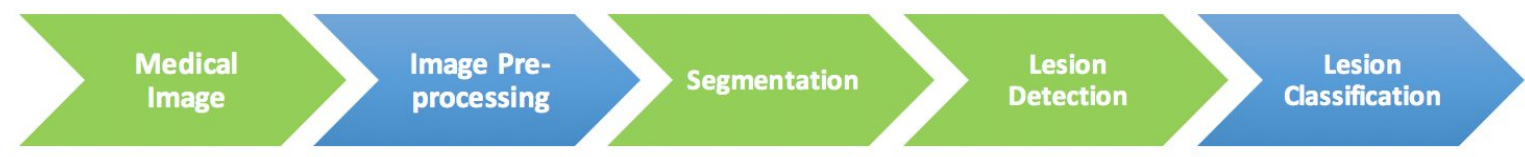

Figure 1. Computer Aided Diagnosis Stages

$\mathrm{BE}$ is the change of normal squamous epithelium in the cells in the lining of the esophagus into metaplasia cells containing goblet cells. ${ }^{7}$ Once BE reaches its highest stage and turns to be cancerous it is considered deadly with very low survival rate. ${ }^{8}$ The transformation of the cells in the esophagus can develop into something called dysplasia that can be divided into several stages from mild, moderate then severe which turns to cancer stage after that. The transformation from one stage to another is considered very small which makes it difficult to detect also the infection can happen in a different region but not all the esophagus tube. The deformation in the cells of an esophageal area can cause a change in the cell structure and vascular appearance to be classified into four histological grades Normal Squamous (NS), Gastric Metaplasia (GM), Intestinal Metaplasia (IM) and neoplasia (NPL). GM is the presence of gastric type mucus secreting cells in the surface epithelium, IM is similar to GM with more mucus with goblet cells, the dysplasia is indicated at pathway of the esophagus and is precancerous. NPL appears when the cells are cancerous. Each of these types has its own cell and vessel architecture. The vessels of GM have a regular shape that appears in deeper parts of the mucosal layer and the cells have a regular shape with a cobblestone appearance. In the case of IM, the epithelium starts to be visible in the upper and deeper part of mucosal layer accompanied with goblet and cylinder dark cells. When reaching the NPL stage the vessels and cells have an irregular appearance with dark contrast. ${ }^{9}$ Confocal Laser Microendoscopy (CLE) is considered one of the latest technologies used for endoscopy examination. ${ }^{10}$ It is an in-vivo endoscopic tool that allows imaging and diagnosis at the same time. It has been accounted especially by non-expert CLE endoscopist that there is an instability in the accuracy results when classifying IM, GM and NPL. ${ }^{11}$ Also, it requires an observer that is very well trained due to the very minor changes that are considered challenging. ${ }^{12}$ Moreover, the physician is required to have the basic knowledge about histopathology to differentiate between normal and abnormal cells.

\section{PROPOSED MODEL}

The presented classification model is divided into three separate phases as shown in figure 3 . The first phase is the enhancement process that involves the implementation of a novel filter is implemented and applied to the image. Thus, improve the internal features on different levels, then various features are extracted according to the cells and vessel structure to be finally classified with a presented multistage classifier.

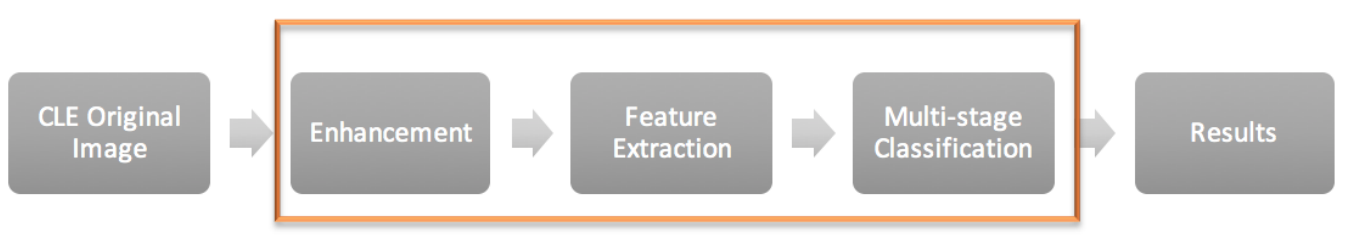

Figure 2. Proposed Model 
Image enhancement aims at reducing the noise in an image and clarifying its concealed details. In the first phase of the proposed model, the features of a CLE image are enhanced by applying a proposed digital filter that utilizes the fractional differential (FD) and fractional integration (FI) in the wavelet sub-bands. As shown in figure 3, the developed filter firstly decomposes the image into its Discrete Wavelet Transform (DWT), resulting in four sub-bands (LL, LH, HL, and HH). Then, FI is applied to the Diagonal sub-bands in order to remove the noise, while the FD is applied to the HH sub-band to improve selected texture features. The improved image is reconstructed by applying the Inverse DWT (IDWT) and then the FD filter is reapplied on the whole reconstructed image to improve the overall texture. The filter is applied in the DWT sub-bands to benefit from the remarkable properties of DWT in feature improvement.

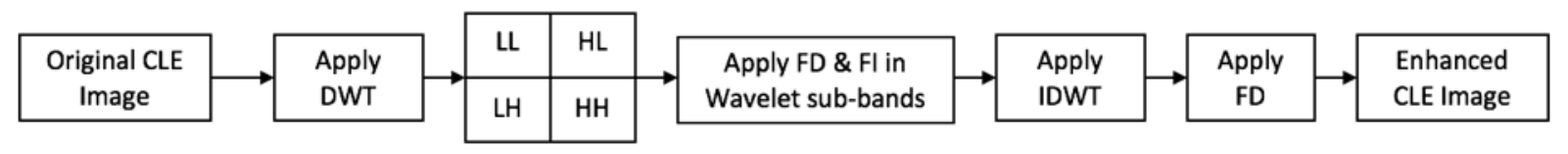

Figure 3. Proposed Enhancement Filter

Feature selection is a key factor in image classification. The selected features chosen to represent target area are considered the main difficulty of obtaining a high accuracy result for CAD system. ${ }^{13}$ Various features are usually combined together to identify an image characteristic since a single feature might fail to represent the details. ${ }^{14}$ An aggregation of texture and intensity features have been extracted aiming to improve the classification results in our proposed model. The features chosen in our proposed model were based on the properties of the histology stage as each grade type has a certain internal structure. The time and computation complexity also need to be considered, as the CLE is done in-vivo and the automatic classification process needs to be in real-time. The following is a list of the main chosen features: Gray Level Co-occurrence Matrices $($ GLCM $),{ }^{15}$ Fractal Texture Features, ${ }^{16}$ Fuzzy Local Binary Patter(FLBP),${ }^{17}$ Intensity Features and Wavelet Features.

Multistage Classification Model is implemented in order to classify between IM, GMP and NPL, where various features have been extracted after enhancing the images, these set of features were divided into three batches and named as follow:

- Feature_Vector_1: Intensity Features,GLCM, Fractal Dimension, Lacunarity.

- Feature_Vector_2: Wavelet Feature Coefficient, GLCM, Fractal Dimension, Lacunarity.

- Feature_Vector_3: Fuzzy LBP,GLCM, Fractal Dimension, Lacunarity.

These set of features batches are grouped as a preparation for categorizing the image into its equivalent class. Figure 4 shows the proposed classification model. In the first stage, the input image is categorized into either NPL or Other. To classify between these two classes, Feature_Vector_1 is used. If the output from this stage is not classified as NPL then the image will be passed to Model 2. Where, Feature_Vector_2 is used to classify the image into GM, IM or Other. Finally, any image that is classified as Other by Model 2, is passed through to Model 3. In Model 3, Feature_Vector_3 is utilized, Accordingly Model 3 classifies the image into IM, GM or NPL.

The features selected in each Feature Vector, for each model, were chosen based on the properties of the images of the types/grades that need to be classified in that model at that current stage. The GLCM and fractal dimension use has been standard in all the feature Vectors used. The reason for this is that the region of dysplasia usually has a high entropy value and a low correlation of pixel pairs. Fractal texture based has the ability to describe texture pattern which will be used as one of the distinctive features to describes the cell property. When classifying the NPL we take into consideration the gray intensity features. The NPL has a heterogeneous and brighter signal intensity due to the leakage vessels and the cells have a black color after being cancerous. After that, we use the wavelet transform with the GLCM and fractal dimension to differentiate between GMP and IM. As mentioned before there is a very high similarity between these two types which makes it difficult to 


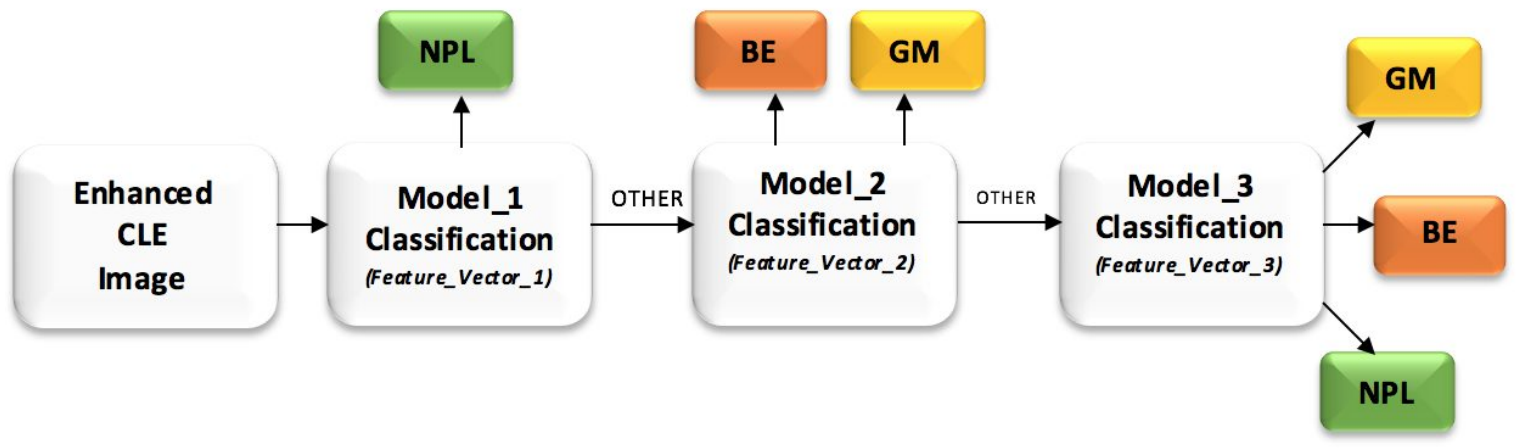

Figure 4. Classification Model

differentiate between the two of them where the BE accompanies goblet cells. Therefore, the internal texture and edge needs to be studied deeply in its wavelet form to have the ability to evaluate the difference between these two types. Finally, the FLBP is extracted for the last phase with the other two features to describe the fuzzy texture patterns and to have the ability to describe imprecise data to discriminate the last image samples left unclassified The classification model started by extracting the NPL class from any other classes as its is the cancerous type that has distinctive properties when compared to the other two grades which provide the system with an improved overall accuracy. As an outcome of the first phase, the images is more likely to be IM or GM if misclassified, for this reason, Model 2 distinguishes between these two types focusing on certain features because as mentioned earlier, they have very similar properties causing difficulty in differentiating between them. Finally, any image that was misclassified till this point will reach the third classifier level to be classified as one of the three histology grades. A pseudo code for the represented classification model is shown below. Experimental results will be presented in the following section to show the classification effectiveness of the presented model.

\section{EXPERIMENTAL RESULTS}

The proposed model has been tested to evaluate the efficiency. The classification was carried on a dataset of 262 images from 32 patients with different histological grades. The process of classification was performed using the Support Vector Machine (SVM). The SVM was utilized using the poly kernel. The leave-one-out approach was utilized to evaluate the classification accuracy. The evaluation of the model is based on classifying between IM, GM, and NPL. But particular emphasize is to increase the detection of IM, since the accurate detection of IM is the most important for early detection and can increase the survival rate and improve early treatment. A sample from each grade from the dataset is shown in Figure 5, before and after enhancement. The proposed enhancement filter was able to improve texture and intensity details within the image. This was also evidenced by the improved classification results.
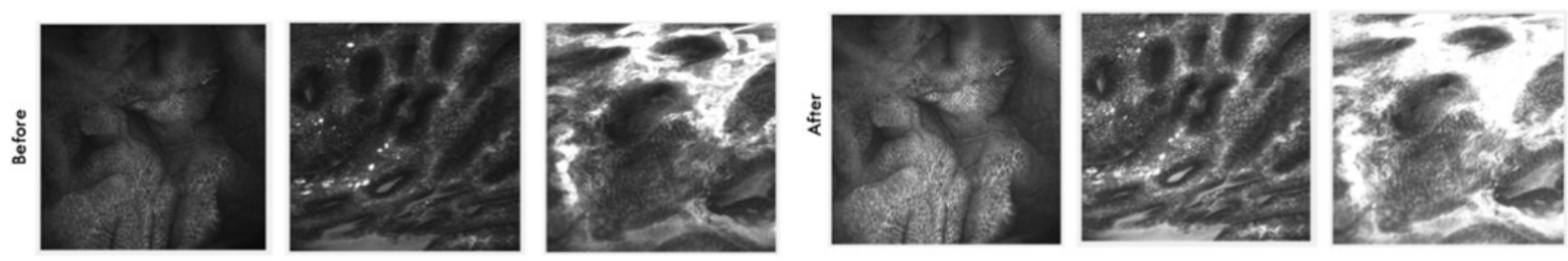

Figure 5. Images Before and After Enhancement

Our main focus in the proposed model is to increase the accuracy of the IM detection as it is considered the main critical precancerous stage. The standard performance measures were used to provide a quantitative 


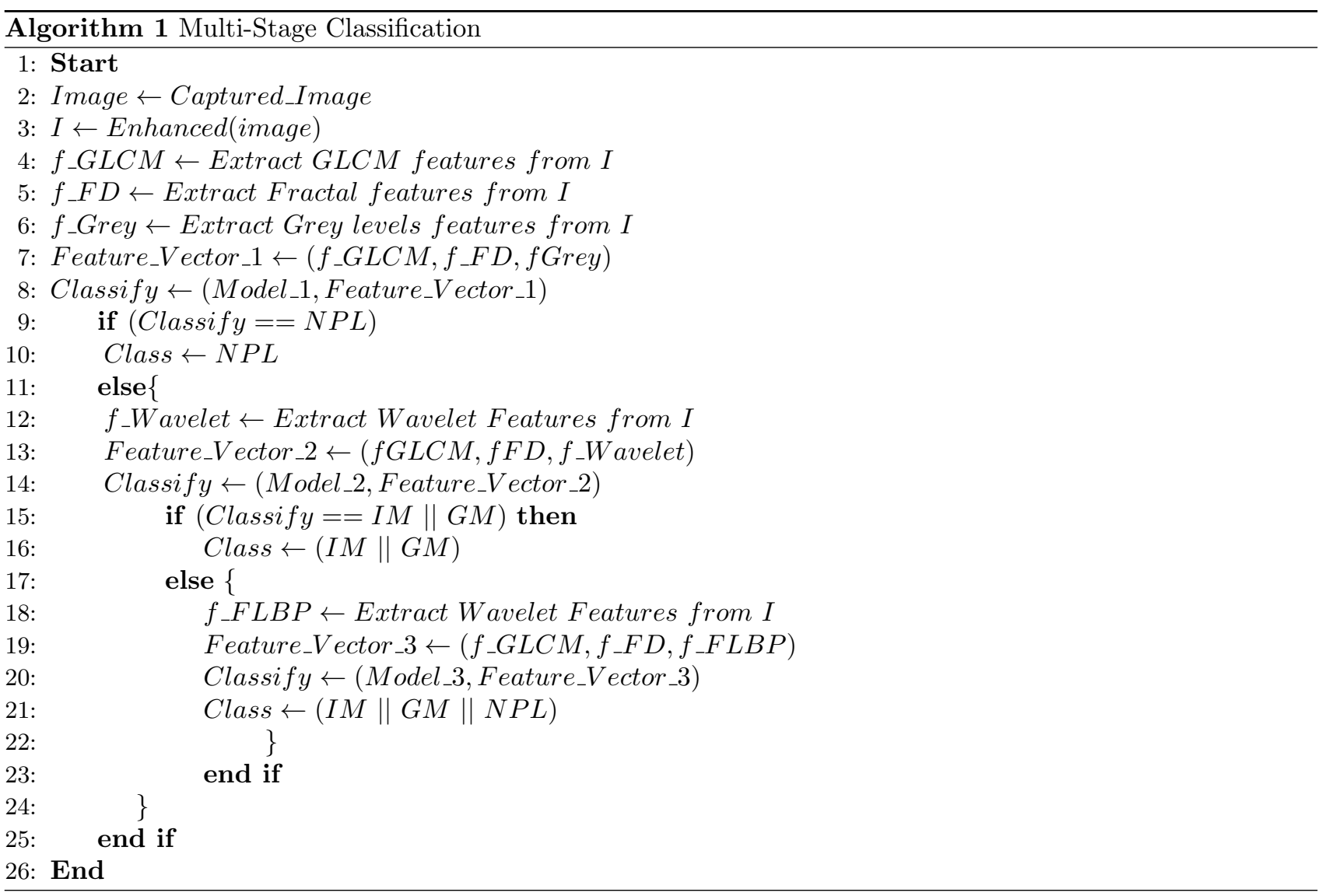

evaluation of the performance. Table I depicts the confusion matrix of the model clarifying the number of images correctly and incorrectly classified per class.

In order to evaluate the performnace of both the enhancement filter and classification model,, Table II represents the results of classification of each category compared with the same model but without exposing the images to the enhancement filter. Also, it's compared with one of the latest baseline models ${ }^{18}$ that relies on extracting the features on an image-based analysis. The proposed model was able to cause an increase of 8-10\% in the overall accuracy compared to the other methods. The proposed enhancement filter caused a significant improvement when applied to the CLE image. The results from the model with the filter were increased in all results accuracy, sensitivity and specificity for the categorize when compared to the same model but without the filter which proves its efficiency. When comparing with ${ }^{18}$ in classifying the IM, the model was able to improve the classification by almost $15 \%$. While when comparing the sensitivity of the other two classes the results of the model proposed were considered comparable but it was able to improve the specificity which signifying that less misleading false positive classification. Both our method and ${ }^{18}$ are image-based feature extraction systems which make that faster and similar in processing time towards the start of the classification process.

In addition to the evaluation performance measures, the timing measure is also a factor that needs to be considered, the reason for this is that the CLE is done in-vivo and the classification needs to be done in realtime. The processing time for each input image from the 262 dataset sample, where each image is exposed to the enhancement filter and then passes through the classification models where the features are extracted and the image is categorized to its histology grade. Figure 6(a) represents the average time taken for each histology type. As represented the IM requires an average of 8.18 seconds, while the GMP took 7.88 seconds and NPL type needed 14.16 seconds to get classified. To get a clearer view about estimating the time, figure $6(\mathrm{~b})$ demonstrates that around $56 \%$ of the dataset needed almost 7.67 seconds and $15 \%$ required 8.43 seconds to be processed. The rest of the images ranged from 9 to 17 seconds. In both cases the time is considered reasonable and convenient 
Table 1. Confusion Matrix of Proposed Model

\begin{tabular}{|c|c|c|c|c|}
\hline & IM & GM & NPL & Sensitivity \\
\hline IM & 160 & 9 & 3 & 0.93 \\
\hline GM & 6 & 21 & 3 & 0.7 \\
\hline NPL & 4 & 0 & 56 & 0.933 \\
\hline Specificity & 0.86 & 0.96 & 0.967 & \\
\hline Accuracy & \multicolumn{5}{|c|}{$90.458 \%$} \\
\hline
\end{tabular}

Table 2. Comparison For Different Histology Stages

\begin{tabular}{|c|c|c|c|}
\hline & Proposed Model & Classification Model Without filter & E.Veronese et. al (2012) \\
\hline & \multicolumn{3}{|c|}{ Accuracy of Grade Classification } \\
\hline & $90.458 \%$ & $80.9 \%$ & $82 \%$ \\
\hline Class & \multicolumn{3}{|c|}{ Sensitivity for Each Class Classification } \\
\hline IM & 0.932 & 0.88 & 0.78 \\
\hline GM & 0.7 & 0.333 & 0.78 \\
\hline NPL & 0.933 & 0.9 & 1.00 \\
\hline Class & \multicolumn{3}{|c|}{ Specificity for Each Class Classification } \\
\hline IM & 0.86 & 0.75 & 0.97 \\
\hline GM & 0.96 & 0.9 & 0.94 \\
\hline NPL & 0.98 & 0.97 & 0.79 \\
\hline
\end{tabular}

in the examination process since the mean inspection time of the CLE is around 22 minutes where a patient need from 9 to 45 minutes to be examined but considered not compatible with the properties of the machine in capturing the image as it captures 0.8 frames per second. ${ }^{3}$

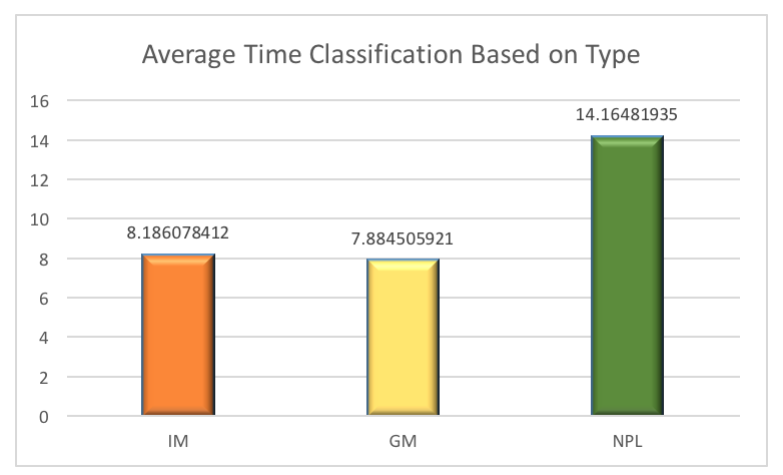

Figure 6. Time for image ratio

A way of measuring the performance of a $2 \mathrm{D}$ dimensional classification is analyzing the Receiver Operating Characteristic (ROC) curve. The process of the first phase allows an option to reduce the false positive related to the IM \& GM as shown in the ROC curve in figure 15(a). Setting the operating point giving the highest accuracy for the single classification node in discriminating between NPL and other images is $98.762 \%$. Similarly, second classifier operating point was analyzed through the ROC curve in figure 15(b) where the True positive represents the accuracy of separating the IM from GM with an accuracy of $96.705 \%$. 

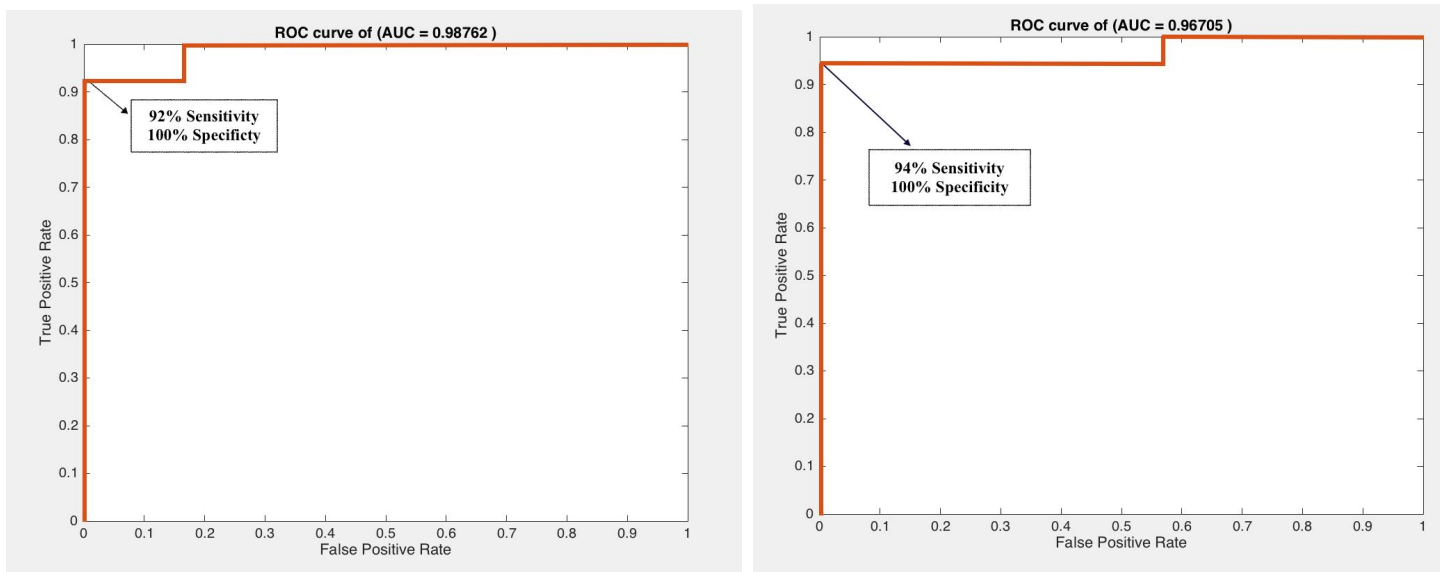

Figure 7. ROC Curve analysis for each Classifier

\section{CONCLUSION}

A proposed computerized method that automatically classifies between different grades of Barretts Esophagus such as Gastric Metaplasia (GM), Intestinal Metaplasia (IM) and neoplasia (NPL), from CLE images has been presented. The main target of the model is to increase the classification accuracy of the IM beside the other types, to assist the doctors in a second opinion before the dyslpia turns into a deadly cancerous and support the decrease of number of biopsy sample needed. The main contribution of the work lies into several stages, first, improving the features of the image by using a proposed filter, then selecting different feature vectors and finally grouping them based on classification stage. This is achieved by applying an enhancement filter that utilizes the FD and FI in the DWT sub-bands of an image then reapplying the FD on the whole reconstructed image, in order to improve the overall texture. Different features were extracted from the enhanced image, based on studying the properties of the cell and vessel architecture of columnar epithelium in its different stages. Finally, these extracted features were used for a multistage classification process where the model was evaluated through the leave-one-out approach.. Results demonstrated the efficiency on all classified grades. The classification was compared with most recent approach where the presented model was able to prove its efficiency in regards to the accuracy, sensitivity and specificity.

\section{REFERENCES}

[1] Boschetto, D., Gambaretto, G., and Grisan, E., "Automatic classification of endoscopic images for premalignant conditions of the esophagus," in [SPIE Medical Imaging], 978808-978808, International Society for Optics and Photonics (2016).

[2] Qi, X., Pan, Y., Sivak, M. V., Willis, J. E., Isenberg, G., and Rollins, A. M., "Image analysis for classification of dysplasia in barrett's esophagus using endoscopic optical coherence tomography," Biomedical optics express $\mathbf{1}(3), 825-847$ (2010).

[3] Kiesslich, R., Gossner, L., Goetz, M., Dahlmann, A., Vieth, M., Stolte, M., Hoffman, A., Jung, M., Nafe, B., Galle, P. R., et al., "In vivo histology of barrett's esophagus and associated neoplasia by confocal laser endomicroscopy," Clinical Gastroenterology and Hepatology 4(8), 979-987 (2006).

[4] Gill, R. S. and Singh, R., "Endoscopic imaging in barrett's esophagus: current practice and future applications," Annals of Gastroenterology 25(2), 89 (2012).

[5] Doi, K., "Computer-aided diagnosis in medical imaging: historical review, current status and future potential," Computerized medical imaging and graphics 31(4), 198-211 (2007).

[6] Qi, X., Sivak, M. V., Isenberg, G., Willis, J. E., and Rollins, A. M., "Computer-aided diagnosis of dysplasia in barrett's esophagus using endoscopic optical coherence tomography," Journal of biomedical optics 11(4), 044010-044010 (2006). 
[7] Conteduca, V., Sansonno, D., Ingravallo, G., Marangi, S., Russi, S., Lauletta, G., and Dammacco, F., "Barrett's esophagus and esophageal cancer: an overview," International journal of oncology 41(2), 414424 (2012).

[8] Buchner, A. M. and Wallace, M. B., "In-vivo microscopy in the diagnosis of intestinal neoplasia and inflammatory conditions," Histopathology 66(1), 137-146 (2015).

[9] Leggett, C. L. and Gorospe, E. C., "Application of confocal laser endomicroscopy in the diagnosis and management of barrett's esophagus," Annals of Gastroenterology 27(3), 193 (2014).

[10] Lim, L. G., Yeoh, K. G., Salto-Tellez, M., Khor, C. J., Teh, M., Chan, Y. H., So, J. B., Rajnakova, A., Shen, E., Srivastava, S., et al., "Experienced versus inexperienced confocal endoscopists in the diagnosis of gastric adenocarcinoma and intestinal metaplasia on confocal images," Gastrointestinal endoscopy 73(6), 1141-1147 (2011).

[11] Rajan, P., Canto, M., Gorospe, E., Almario, A., Kage, A., Winter, C., Hager, G., Wittenberg, T., and Münzenmayer, C., "Automated diagnosis of barrett's esophagus with endoscopic images," in [World Congress on Medical Physics and Biomedical Engineering, September 7-12, 2009, Munich, Germany], 21892192, Springer (2009).

[12] Naini, B. V., Chak, A., Ali, M. A., and Odze, R. D., "Barrett's oesophagus diagnostic criteria: endoscopy and histology," Best Practice 83 Research Clinical Gastroenterology 29(1), 77-96 (2015).

[13] Liu, D.-Y., Gan, T., Rao, N.-N., Xing, Y.-W., Zheng, J., Li, S., Luo, C.-S., Zhou, Z.-J., and Wan, Y.-L., "Identification of lesion images from gastrointestinal endoscope based on feature extraction of combinational methods with and without learning process," Medical image analysis 32, 281-294 (2016).

[14] Cong, Y., Wang, S., Liu, J., Cao, J., Yang, Y., and Luo, J., "Deep sparse feature selection for computer aided endoscopy diagnosis," Pattern Recognition 48(3), 907-917 (2015).

[15] Haralick, R. M., "Statistical and structural approaches to texture," Proceedings of the IEEE 67(5), 786-804 (1979).

[16] Costa, A. F., Humpire-Mamani, G., and Traina, A. J. M., "An efficient algorithm for fractal analysis of textures," in [2012 25th SIBGRAPI Conference on Graphics, Patterns and Images], 39-46, IEEE (2012).

[17] Iakovidis, D. K., Keramidas, E. G., and Maroulis, D., "Fuzzy local binary patterns for ultrasound texture characterization," in [International Conference Image Analysis and Recognition], 750-759, Springer (2008).

[18] Grisan, E., Veronese, E., Diamantis, G., Trovato, C., Crosta, C., and Battaglia, G., "239 computer aided diagnosis of barrett's esophagus using confocal laser endomicroscopy: Preliminary data," Gastrointestinal Endoscopy 75(4), AB126 (2012). 